\title{
Freezing of spherical droplets in the environment of cold air
}

\begin{abstract}
The problem of spherical droplets freezing in a stream of cold air is considered. Generalized equation for finding the depth of freezing of the spherical layer depends on time and thermo physical properties of the fluid was proposed. The full period time of spherical layer freezing consists of two parts: the first is preliminary cooling of droplet down to the phase change temperatures and the second period connects with freezing spherical layer time. The description of the experimental installation and the obtained experimental data were presented. The theoretical generalization was conformed with the theory.
\end{abstract}

Keywords: droplet, solid phase, freezing, fourier differential equation, boundary condition, refrigeration plant, cylindrical channel, water-saturated droplet, regular law
Volume 3 Issue I - 2018

\author{
Marinyuk BT, Ugolnikova MA, Zabugina AV \\ Department of Low Temperatures Engineering, Moscow \\ Polytechnic University, Russia
}

Correspondence: Marinyuk BT, Department of Low Temperatures Engineering, Moscow Polytechnic University, Russia, Email MarinykB@yandex.ru

Received: January 09, 2018 | Published: February 15, 2018

\section{Introduction}

Production of natural hydrocarbon, transportation and filling into storage is often carried out in the Northern regions and is accompanied by direct contact of droplets produced with the environment cold air. The droplets initially may have a positive temperature. A similar problem occurs when the final product of some chemical substances have liquid - droplet state, the conditions of preservation of them demands solidification in a low temperature environment. In particular, this process is carried out in a stream of cold air. Advantages of this technology is obvious, if the liquid-droplets substances prone to decay or have high volatility. Heat treatment of such substances allows to preserve the integrity of the product and increases the safety of storage and of working with them.

In the existing literature there are publications on this problem, which suggest solutions often difficult to implement because they require special programs for obtaining numerical results, in addition, some of them consider the object of freezing is initially chilled to a temperature of phase transition of a substance in the solid state. ${ }^{1}$

\section{Theory}

Consider a spherical droplet, with a fixed radius $r$, the properties of liquid substance: - heat capacity $\mathrm{C}_{\mathrm{p}}$ thermal conductivity $\lambda$ of ice are known as well as the parameters of the air environment surrounding the droplet, temperature air $\mathrm{T}_{\mathrm{a}}$, air velocity, its thermal properties. $\mathrm{Tw}_{\mathrm{i}}$.

The initial temperature droplet is fixed and has a positive value

To solve the task the following assumptions are accepted:

i. Geometrical dimensions of the droplet do not change in the process of cooling and freezing.

ii. Thermo physical properties of liquid and solid phases of a substance in the heat exchange process remain constant.

iii. The intensity of heat transfer over the surface droplet is the same.

iv. The air temperature does not change with time. v. The law of heat transfer down to the stadium of to the phase transition is close to regular.

The scheme of interaction of the droplet-s with the cold flow air is demonstrated in Figure 1.,2

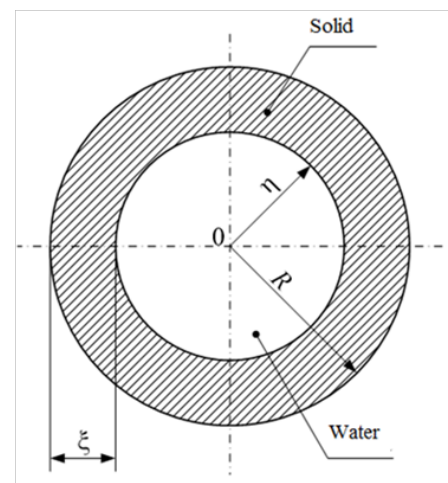

Figure I The flow sheet of interaction of the droplets with the cold air.

Fourier differential equation of heat conduction transfer for a spherical layer is:

$$
\frac{1}{a} \cdot \frac{\partial T}{\partial \tau}=\frac{\partial^{2} T}{\partial r^{2}}+\frac{2}{r} \cdot \frac{\partial T}{\partial r}
$$

Where a is the temperature diffusivity for the solid phase layer, $\mathrm{m} 2 / \mathrm{s}^{3}$

Boundary conditions have the form:

$$
T(R, \tau)=T_{0}
$$

Where $\mathrm{T}_{0}$ is a variable of droplet surface temperature, $\mathrm{K}$.

Further,

$$
\begin{aligned}
& T(\mathrm{r}, 0)=T_{w i} \\
& T(\eta, \tau)=T_{f}
\end{aligned}
$$


Where $T_{w_{i}}$ and $\mathrm{T}_{\mathrm{f}}$ - initial temperature of the liquid (more 273K) and the temperature of the phase transition of the liquid to the solid phase, respectively K.

Thermal condition at the boundary of solid phase -water:

$$
-\left.\lambda \cdot \frac{\partial T}{\partial r}\right|_{r=\eta}=\rho \cdot L \cdot \frac{d \eta}{d \tau}
$$

Where $\lambda_{\mathrm{s}}$ is the thermal conductivity of the solid phase, $\lambda_{\mathrm{s}}=2.3$ $\mathrm{W} / \mathrm{m} \cdot \mathrm{K} .^{4}$

$\rho$ is the density of the solid phase, $\mathrm{kg} / \mathrm{m} 3$

$\mathrm{L}$ is the heat of phase transition of liquid to solid phase, $\mathrm{j} / \mathrm{kg}$

$\tau$ - Time of the process, $\mathrm{c} \mathrm{r}$ and $\eta$ is the current coordinate in the layer of the solid phase and the coordinate of the front phase transformation, respectively.

To solve the problem, we introduce a generalized variable $v$

$v=\frac{R-r}{\sqrt{\tau}}$

Accordingly, when $\mathrm{r}=\eta$, the value of $v$ will turn in to

$$
\beta=\frac{R-\eta}{\sqrt{\tau}}
$$

Boundary conditions 2,3,4 and 5 will take the form

$$
\begin{aligned}
& T(B)=T_{f} \\
& T(\infty)=T_{w i} \\
& T(0)=T_{0}
\end{aligned}
$$

Taking into account the expression (6), the conditions on the boundary of the liquid - solid phase takes the form

$$
\begin{aligned}
& \frac{-\lambda_{s}}{\sqrt{\tau}} \frac{d T}{d v} \mid v=\beta=\rho \cdot L \cdot \frac{\beta}{2 \sqrt{\tau}} \\
& \frac{-\lambda_{s}}{\sqrt{\tau}} \frac{d T}{d v} \mid v=\beta=\rho \cdot L \cdot \frac{\beta}{2 \lambda_{s}}
\end{aligned}
$$

Evaluation of the surface temperature droplet $\mathrm{T}_{0}$ can be obtained on the basis of the of prof. Leibenzon's method, which assume stationary temperature distribution within the spherical layer of the solid phase. $\mathrm{T}_{0}$.

Omitting intermediate transformations, we find the expression for

$$
T_{0}=\frac{\frac{\propto_{a} R T_{a}}{\lambda_{s} \eta}+\frac{T_{f}}{R-\eta}}{\frac{1}{R-\eta}+\frac{\propto_{a} R}{\lambda_{s} \eta}}
$$

By substituting the expression (6) into equation (1), we obtain the differential equation in full derivatives

$$
\frac{-1}{a} \cdot \frac{d T}{d \nu} \cdot v \sqrt{\tau} \cdot \frac{1}{2} \cdot \tau^{\frac{-3}{2}}=\frac{1}{\sqrt{\tau}} \cdot \frac{d^{2} T}{d \nu^{2}}+\frac{2 \cdot a}{v \cdot \sqrt{\tau}+\eta} \cdot \frac{d T}{d \nu} \cdot \frac{1}{\sqrt{\tau}}
$$

Equation (14) can be solved by decomposition it into a power series. $^{5}$

$$
T(v)=T_{0}+T_{v=\beta}^{\prime} \cdot \frac{v-\beta}{1 !}+T_{v=\beta}^{\prime \prime} \cdot\left(\frac{v-\beta}{2 !}\right)^{2}+\ldots T_{v=\beta}^{n} \cdot \frac{v-\beta}{n !}
$$

Substituting the appropriate values for $T_{0}, T_{v=\beta}^{\prime}$ and $T_{v=\beta}^{\prime \prime}$ and applying the condition ten one can receive

$$
T_{f}=\frac{\frac{\propto_{a} R T_{a}}{\lambda_{s} \eta}+\frac{T_{f}}{R-\eta}}{\frac{1}{R-\eta}+\frac{\propto_{a} R}{\lambda_{s} \eta}}+\frac{\rho L \cdot\left(\frac{R-\eta}{\sqrt{\tau}}\right)^{2}}{2 \lambda_{s}}+\frac{\rho L \cdot\left(\frac{R-\eta}{\sqrt{\tau}}\right)^{3}}{4 \lambda_{s}} \cdot\left(\frac{2 \sqrt{\tau}}{\eta}\right)
$$

Returning to expression (12), we note that the intensity of heat transfer from the liquid to the interface solid phase within the droplet is not possible to determine, therefore, we assume that the mode of heat transfer occurs according to the regular law. Then the cooling time of the droplet is expressed as

$$
\tau=\frac{M C_{p_{w}}}{a_{a} F} \ln \frac{T_{w_{i}}-T_{a}}{T_{w}-T_{a}}
$$

Where $\mathrm{M}$ is the mass of droplet, $\mathrm{kg}$

$\mathrm{Cp}_{\mathrm{w}}$ - specific heat of fluid, $\mathrm{j} / \mathrm{kg} \mathrm{K}$

$\alpha_{\mathrm{a}}$ - heat transfer coefficient from the air to the droplet surface, $\mathrm{W} / \mathrm{m} 2 \mathrm{~K}$

$\mathrm{F}$ - The droplet surface, $\mathrm{m}^{2}$

$\mathrm{T}_{\mathrm{w}}$ - current temperature of the droplet, in which in the limit takes it is phase transition temperature, $\mathrm{K}$

$\mathrm{T}_{\mathrm{a}}$ - temperature of air flow, $\mathrm{K}$.

The calculation plan is that at the beginning the temperature droplet time is determined while it is cold down to phase change state by the equation (17), $\tau 1$.

Further, on the basis of the solution of equation (16), we define the time of freezing of droplet spherical lay to a depth of $R-\eta, \tau 2$. The total solidification time to a depth $\mathrm{R}-\eta$ within the droplet will be $\tau=$ $\tau 1+\tau 2$.

\section{Experimental}

To verify the above relationships, an experiment on the freezing of water-saturated and glycerol spherical bodies in a stream of cold air were conducted. Figure 2 shows a scheme of the experimental stand that includes a cooling chamber 1 with a refrigerating unit 2 and the cooler 3, camera placed inside the vertical channel 4 is fed by the discharge fan 5, air flow control.

The experiments were conducted under the following conditions:
i. Air temperature $\mathrm{Ta}=-15^{\circ} \mathrm{C}(258 \mathrm{~K})$
ii. Object of study: water-saturated spherical object $\mathrm{d}=12 \mathrm{~mm}$
iii. The phase transition temperature of $\mathrm{Tf}=273 \mathrm{~K}$
iv. Coefficient of thermal conductivity of the solid phase $\lambda \mathrm{s}=2,3$ $\mathrm{W} / \mathrm{mK}$ 
v. The heat transfer coefficient of air, $\alpha=90 \mathrm{~W} / \mathrm{m} 2 \mathrm{~K}$

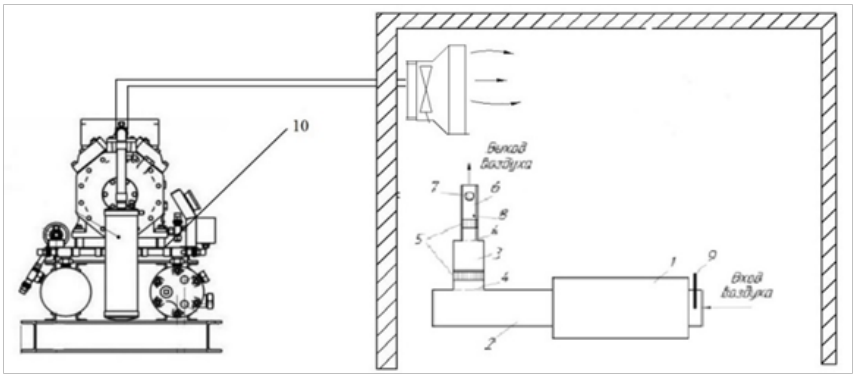

Figure 2 The scheme of experimental installation: I - fan; 2 - channel of air flow stabilization; 3 - cylindrical channel; 4 - grid; 5 - mesh partition; 6 working channel; 7 - an experienced element; 8 - thermocouple temperature sensor; 9 - latch; 10 - compressor-condensation unit.

\section{Estimation of the error of measuring instruments}

When conducted the experiments, the following values has been determered: temperature, air flow rate, time, diameter and thickness of the obgect as well as its weight. Let us estimate the instrument error when measuring these values. Temperature sensors were used. They were attach with digital receiver temperature transmitter (Figure 3) providing a range of measured temperatures from minus 50 to plus $200^{\circ} \mathrm{C}$ with an accuracy of $0.1^{\circ} \mathrm{C}$. Maximum absolute error of temperature measurement is $\delta t_{\max }=0.5 \%$.

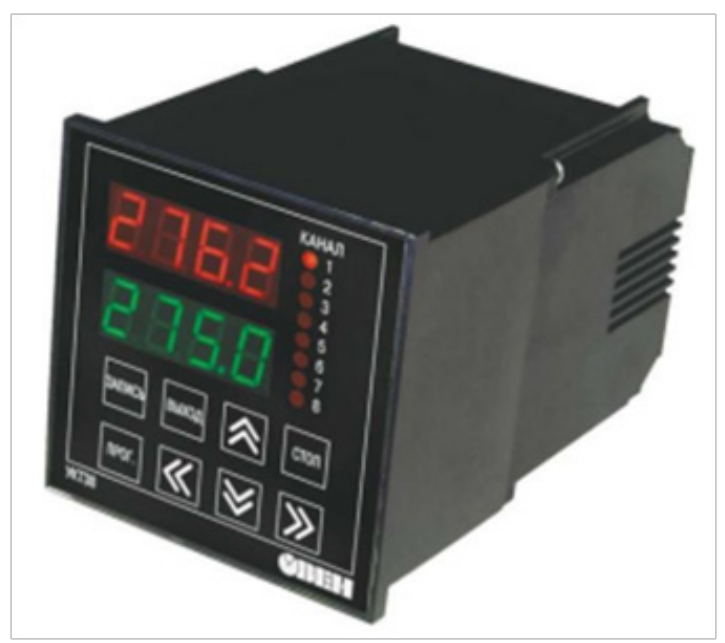

Figure 3 Digital receiver temperature transmitter.

Measurement of the rate of air flow was conducted using an anemometer Aero Temp x-line (Figure 4). According to the passport of the device measuring range of the device $0-30 \mathrm{~m} / \mathrm{s}$, accuracy $\delta \max =3 \%$.

Diameter and thickness of frozen layer were measured with the help a digital caliper (Figure 5), it is equipped with an electronic reading device and allows to obtain the output data with high accuracy (Table 1).

\begin{tabular}{|l|l|l|l|}
\hline Name & Measuring range & Division value & Accuracy, $\delta \mathbf{m a x}$ \\
\hline Caliper device & $0-150 \mathrm{~mm}$ & $0,01 \mathrm{~mm}$ & $\pm 0,03 \mathrm{~mm}$ \\
\hline
\end{tabular}

The time of the experiment was measured using an electronic stopwatch. The error of time measurement in a process that takes more than 10 minutes is less than $1 \%$. The mass of the object was measured on the laboratory electronic scales ACOM JW-1 (Figure 6). According to the passport of the device absolute error in the measurement of time is $\delta$ max $=0.1 \mathrm{~g}$.

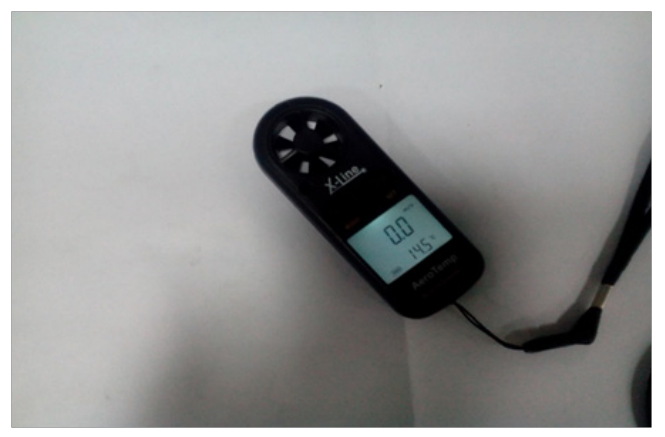

Figure 4 Aero Temp $x$-line anemometer.

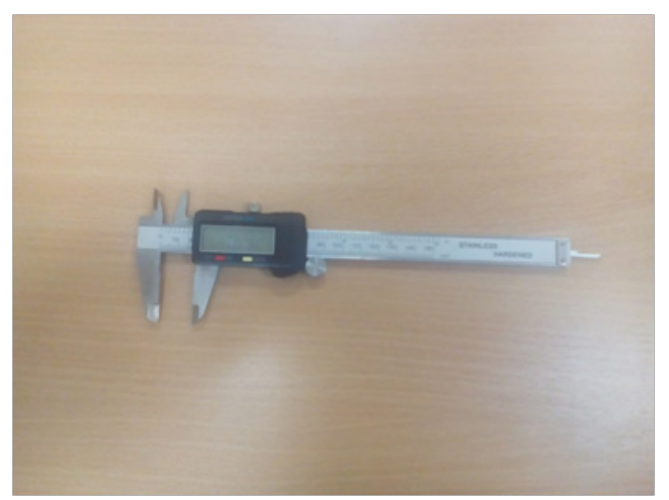

Figure 5 Digital caliper.

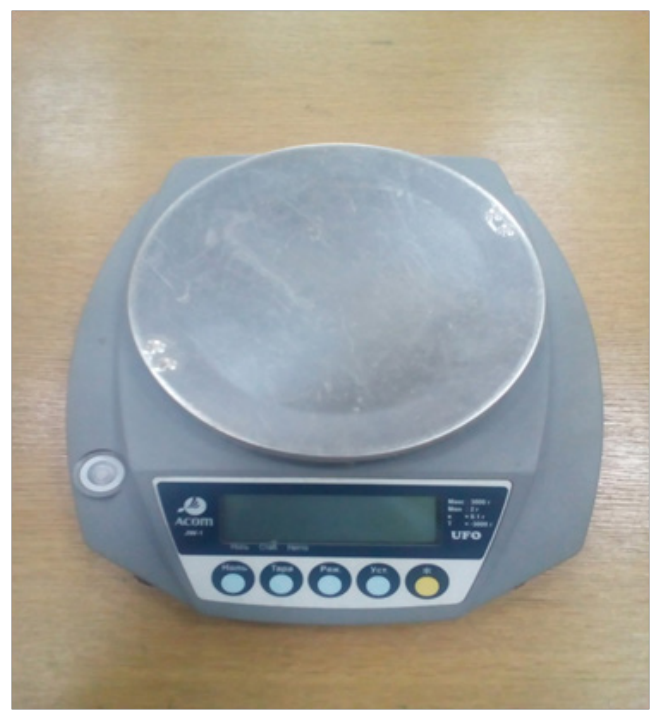

Figure 6 Laboratory electronic scales ACOM JW-I.

\section{Experimental procedure}

Chilled in the refrigerator, the air passing through the fan (1) is supplied to the stabilization channel (2). In a cylindrical channel (3) droplets levitated in a stream of cold air flowing over the surface of the them. The prose goes on at the beginning of the cooling down to sphere layer solidification. The depth of freezing of a spherical liquid layer is measured after the experiment was finished being done in the environment of the cold air chamber. 


\section{The Results of experiment}

Figure 7 shows the dependence of the relative thickness of the freezing layers within droplets.

i. water-saturated sphere of diameter $\mathrm{d}=12 \mathrm{~mm}$ experiment and theory

ii. a spherical droplet of glycerol $\mathrm{d}=6 \mathrm{~mm}$ calculated data.

iii. As can be seen from the figure 3 the theoretical and experimental results are in a good agreement.

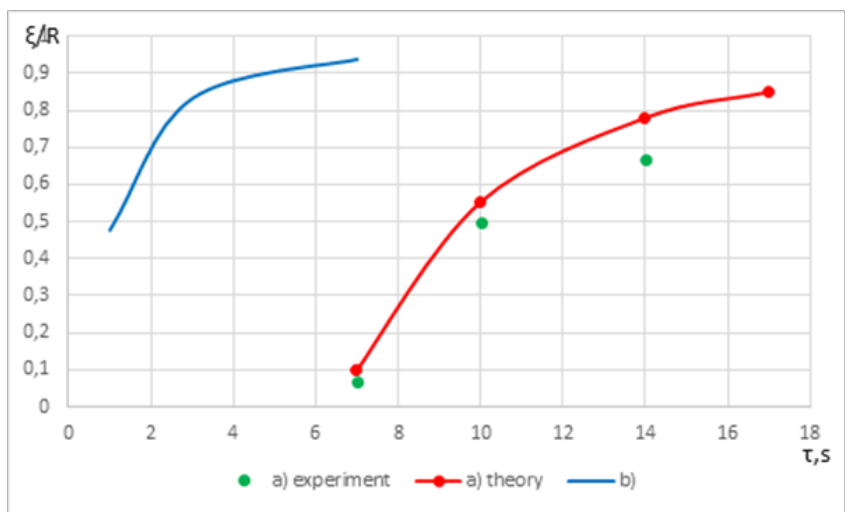

Figure 7 The dynamics of freezing of spherical droplets.

\section{Conclusion}

a. The problem of spherical droplets freezing in a stream of cold air is considered. b. Generalized equation for finding the depth of spherical layer freezing was suggested. It depends on time and thermo physical properties of the substances.

c. The full freezing layer period of time consists of two parts: the first is preliminary cooling of droplet down to the phase change temperatures, the second period connects with freezing spherical layer time.

d. The description of the experimental installation and the obtained experimental data conformed the theoretical generalization.

\section{Acknowledgments}

None.

\section{Conflict of interest}

The author declares no conflict of interest.

\section{References}

1. Olcer NY. Unsteady Temperature Distribution In A Sphere Subjected To Time Dependent Surface Heat Flux And Internal Heat Source. J Heat Transfer. 1969;91(1):45-50.

2. Luikov AV. Theory of Heat Conductivity. 1967.

3. Carslaw HS. Conduction of Heat in Solids. Oxford, UK, 1959.

4. Dean JW, Timmerhaus KD. Thermal conductivity of solid H2O and D2O at low temperatures. Adv in Cryogen Engineering. 1963;8:263-267.

5. Marinyuk BT. The Calculation Of Heat Transfer. In the apparatus and the low-temperature cooling systems. Mashinostroenie. Russia, 2015. 